\title{
Intento de Suicidio Vía Ingesta de Fármacos en Niños
}

\author{
BETTINA VON DESSAUER G. ${ }^{1}$, PAULA ORTIZ F. ${ }^{2}$, TAMARA HINOSTROZA C. ${ }^{3}$, \\ ALEXANDER BATASZEW VD. ${ }^{4}$, ALEJANDRO VELÁSQUEZ D. ${ }^{3}$, \\ MAGDALENA CORREA P. ${ }^{5}$, JAVIERA CÁCERES M. ${ }^{3}$, ÁLVARO MULET B. ${ }^{3}$ \\ Unidad de Paciente Crítico Hospital Roberto del Río, Universidad de Chile, Santiago, Chile. \\ 1. Médico Jefe Unidad de Paciente Crítico Pediátrico Hospital Roberto del Río. \\ 2. Médico Residente Unidad de Paciente Crítico, Hospital Roberto del Río. \\ 3. Médicos en programa de formación en Pediatría, Hospital Roberto del Río, Universidad de Chile. \\ 4. Médico General, Hospital Padre Hurtado. \\ 5. Medico en Formación Universidad de Chile, Psiquiatría en Salud Mental, Hospital Roberto del Río.
}

\begin{abstract}
Suicide attempts secondary to self-inflicted drug poisoning

Suicide attempts in children are an important worldwide cause of morbidity and mortality and an emerging reason for admission in Pediatric Critical Care. Objective: Identify the epidemiologic and clinical characteristics of patients with drug poisoning with a suicide purpose as an admission cause in a Chilean Pediatric Intensive Care Unit (PICU). Design: Retrospective observational study. Setting: 14 beds PICU of a tertiary care public teaching hospital in Santiago de Chile. Material and Method: All patients admitted to the PICU between January 2005 and December 2008 for self-inflicted drug poisoning with an expressed intention of suicide were included. Analysis of the PICU clinical chart was performed with sampling on an excel data base. For seasonal distribution we used statistical analysis Xi2. Results: 178 of 3045 PICU admissions were diagnosed as drug poisoning $(5,8 \%), 108$ of them for suicide attempts $(60,6 \%)$. The number of annual patients increased, with highest incidence in spring and autumn and lowest in winter. Mean and median age was 13 years, $8,4 \%$ less than 12 years. $88,9 \%$ were females. They used polipharmacy in $57 \%$. Drugs were taken from home. Antidepressants and sedatives were the most often used. An underlying psychiatric condition was present in 100 cases $(92,6 \%)$. Associated factors were family disfunction, school problems, abuse and violation and bullying. Mean PICU stay was 1,46 days with $16,7 \%$ requiring transient mechanical ventilation No death was reported in this serie. Conclusions: The number of cases of suicide attempt via drug ingestion as a motive for admission in PICU is increasing, causing a raising number of short and transient admissions, more in spring less in winter. Risk factors were mostly an underlying psychiatric disorder and family disfunction.
\end{abstract}

(Key words: Suicide attempt, drug poisoning, risk factors, PICU, children).

Rev Chil Pediatr 2011; 82 (1): 42-48

Trabajo recibido el 01 de octubre de 2010, devuelto para corregir el 03 de diciembre de 2010, segunda versión el 31 de enero de 2011, aceptado para publicación el 07 de febrero de 2011.

Correspondencia a:

Dra. Bettina Von Dessauer G.

E-mail: bvd@mi.cl 


\section{RESUMEN}

Introducción: El intento de suicidio en pediatría, via ingestión de fármacos, es una importante causa de morbimortalidad en el mundo y un creciente motivo de ingreso a cuidados intensivos. Objetivo: Identificar las características clínicas y epidemiológicas de los pacientes con intoxicación por fármacos con un intento de suicidio, como causa de ingreso a una Unidad de Cuidados Intensivos Pediatricos en Chile (UCIP). Diseño: Estudio retrospectivo observacional. Pacientes y Método: UCIP de 14 camas críticas en un hospital pediátrico de Santiago. Se incluyeron todos los pacientes ingresados a la UCIP debido a intento de suicidio por ingestión de fármacos, entre enero 2005 a diciembre 2008. Resultados: 178 de 3045 egresos se debieron a intoxicación por fármacos $(5,8 \%), 108$ de ellos por intento de suicidio $(60,6 \%)$. La tendencia observada es a un incremento anual, con mayor incidencia en primavera y otoño, y menor en invierno. La media y mediana de edad fue de 13 años, 8,4\% menor de 12 años. 88,9\% fueron mujeres, Se utilizó polifarmacia en un 57\% con fármacos disponibles en el hogar. Antidepresivos y sedantes fueron los más usados. Patología psiquiátrica de base se diagnosticó en un 92,6\% de los casos. Factores asociados fueron disfunción familiar, problemas en el colegio, abuso y violación, bullying. La estadía promedio en UCIP fue de 1,5 días, requiriendo un 16,7\% ventilación mecánica transitoria. No hubo fallecidos en esta serie. Conclusiones: El intento de suicidio por ingestión de fármacos como motivo de ingreso a UCIP ha aumentado, requiriendo estadías cortas y transitorias, mayormente en primavera y menos en invierno. Factores asociados fueron patología psiquiátrica de base y disfunción familiar. (Palabras clave: Intento suicidio, intoxicacion por fármacos, factores riesgo, UCIP, niños).

Rev Chil Pediatr 2011; 82 (1): 42-48

\section{Introducción}

Los intentos de suicidio son un problema mundial reportado desde hace cien años, variando la frecuencia y el motivo en los diferentes grupos etáreos ${ }^{1-5}$. Son más frecuentes en adolescentes, adultos jóvenes, ancianos y mujeres. El suicidio es la tercera causa de muerte entre los adolescentes en EEUU' ${ }^{2,6-8}$.

Entre los agentes empleados en pediatría para el intento de suicidio están los combustibles y el fuego (quemaduras), fármacos y ahorcamiento, lo que en parte depende de factores culturales locales. La relación entre el intento y el logro, la autoeliminación, es de 8:1. Alrededor del $30 \%$ tiene un segundo intento durante el primer año ${ }^{1}$. Múltiples intentos constituyen un riesgo mayor a lograr el objetivo. Estos pacientes tienen un mejor tiempo de planificación y lamentan su recuperación más frecuentemente ${ }^{9,10}$.

Los factores asociados a la ideación suicida y al intento son múltiples. Entre ellos desordenes psiquiátricos preexistentes, victimización y bullying (matonaje) $)^{2,7}$, depresión aguda, estrés, disfunción familiar, obesidad, problemas escolares, pérdida de una relación romántica, fallecimiento inesperado de un cercano, abuso de alcohol o drogas ${ }^{11}$. En EEUU el matonaje tiene una tasa de incidencia de $10-13 \%$ para el victimario y la víctima, y es más frecuente en hombres, con una tasa de depresión asociada mayor que entre los adolescentes sanos. El riesgo suicida no es directamente dependiente de la frecuencia ni de la severidad del matona$\mathrm{je}^{7,12-14}$.

El suicidio en EEUU, según reportes de la JAACAP 2001 Guidelines, es más frecuente en hombres que en mujeres, pero mayor en niñas que en niños ${ }^{2}$.

Hay diferencias en las tasas de suicidio entre países y grupos étnicos, parcialmente atribuible a diferencias locales y culturales, pero también a contagio ${ }^{8,11,13}$.

En Chile, un país en vías de desarrollo, aún existen grandes inequidades en las posibilidades de desarrollo de la juventud, a pesar de la garantía estatal de acceso a la educación y salud como un derecho básico. Estadísticas del MINSAL muestran que la depresión es una condición prevalente en la población chilena con 75/1 000 personas sobre los 15 años. Las principales causas de muerte en adolescentes son externas con un aumento notorio de los suicidios, incluyendo al grupo preadolescente entre los 10-14 años entre el 2000 al 2005. En 
1997, para el grupo entre 10 y 19 años, la tasa de mortalidad por suicidio era de 5,4/100 000 aumentado significativamente hacia el 2000 a $8,6 / 100000$, con relativa estabilización posterior. Alerta el hecho del aumento mayor en hombres ${ }^{15}$.

Información no publicada del 2006 del Servicio de Salud Mental de nuestro hospital, ya revelaba que los agentes más utilizados para el intento de suicidio fueron la ingestión de fármacos y el ahorcamiento. Otro estudio chileno de 42 casos mostró una incidencia de 6,8/1 000 del total de admisiones al hospital entre el 2002-2004, 88\% niñas, con una media de 13,1 años, $53 \%$ usó una combinación de drogas, $49 \%$ tenía una explícita ideación suicida. Los factores de riesgo asociados más relevantes fueron violencia intrafamiliar y depresión. El requerimiento de servicio sanitario global no crítico fue el motivo de admisión al hospital ${ }^{3}$.

Un estudio chileno recién publicado muestra una persistente elevada tasa de ideación de $60 \%$ en adolescentes de 14-20 años. Un tercio de ellos realiza el intento. La frecuencia es mayor en mujeres, colegios públicos y entre los 15-16 años. No se analizan otros factores ${ }^{16}$.

El problema sanitario planteado es de alto impacto pero difícil resolución por sus características y factores sociales relacionados ${ }^{17-21}$.

\section{Hipótesis}

La alta prevalencia de desordenes psiquiátricos en Chile, asociado a problemas sociales de un país en vías de desarrollo, son un escenario propicio para la ocurrencia de intentos suicidas en niños y adolescentes.

\section{Objetivo}

Identificar las características clínicas y epidemiológicas de los pacientes con intoxicación por fármacos con un intento de suicidio como causa de ingreso a una Unidad de Cuidados Intensivos Pediátricos en Chile (UCIP).

\section{Pacientes y Método}

Estudio descriptivo, observacional, retrospectivo. Se incluyeron todos los pacientes ingresados a la UCIP con diagnóstico de in- toxicación por fármacos e intento de suicidio, entre enero 2005 a diciembre 2008. El criterio de ingreso fue sobredosis de fármacos grave con requerimiento de monitorización continua o sustitución de funciones vitales básicas por riesgo vital secundario a compromiso de conciencia, arritmias, inestabilidad hemodinámica o respiratoria, vía aérea inestable, convulsiones u otros con necesidad de hospitalización en UCIP.

Los datos fueron obtenidos de la ficha clínica individual de seguimiento, propia de la UCI, y de la general hospitalaria, y recolectados en una base de datos Excel. Se realizó estudio estadístico $\chi^{2}$ para distribución estacional.

Se definió como intoxicación por fármacos e intento suicida a todo paciente ingresado a la UCIP con la explicitada intención de suicidio por medio de la ingesta voluntaria de uno o más fármacos de cualquier tipo. Disfunción familiar se definió desde la perspectiva de una familia que no brinda el apoyo mínimo al menor para proveer protección: factores relatados fueron detallados como la ausencia sentida de figura materna o paterna debido a separación, suicidio, muerte natural o prisión, abuso de alcohol o sustancias psicoactivas, violencia, maltrato o abuso sexual o desorden psiquiátrico. Polifarmacia se definió como el uso de dos o más fármacos diferentes para el mismo episodio.

\section{Resultados}

Ciento setenta y ocho de los 3045 egresos de la UCIP tuvieron como diagnóstico intoxicación por fármacos $(5,8 \%)$. Sesenta y ocho pacientes (todos menores de 5 años) fueron por ingesta accidental, 2 por parricidio frustrado y 108 por intentos suicidas $(60,6 \%$ de las intoxicaciones). Sólo estos últimos casos fueron incluidos para análisis posterior. Los pacientes fueron admitidos para monitorización en UCIP con un inicial desconocido o impreciso detalle del fármaco usado o de la cantidad ingerida, la mayoría con depresión sensorial y riesgo de depresión respiratoria.

El número de casos por cada 1000 egresos hospitalarios y de la UCIP fue de 0,1/1 000 y 
25,6/1 000 respectivamente. La tendencia observada fue a un incremento anual de pacientes que requieren ingreso a UCIP debido a intento suicida e intoxicación (figura 1).

Las diferencias estacionales fueron estadísticamente significativas, con mayor incidencia en primavera $(p<0,05)$ y otoño, y menor en invierno (figura 2$)$.

En 75 pacientes $(69,4 \%)$ fue el primer intento, en $33(30,6 \%)$ su segundo o más episodio (hasta 5). La mayoría fue en niñas (30 de 33 casos).
La edad media y mediana fue de 13 años (rango 7 a 16 años), 8,4\% tenían menos de 12 años, $90,7 \%$ entre 12 y 16 ; $88,9 \%$ sexo femenino.

Polifarmacia fue el modo elegido en 57\%. Los fármacos más utilizados fueron sedantes (49\%), antidepresivos (45\%), analgésicos y antiinflamatorios $(12,9 \%)$ y otros $(44 \%)$. El antidepresivo más ingerido fue la amitriptilina $(53 \%)$ y entre los sedantes las benzodiazepinas $(41 \%)$ (tabla 1). Es importante remarcar que el Paracetamol aparece en 2008 ( 8 pacientes) con
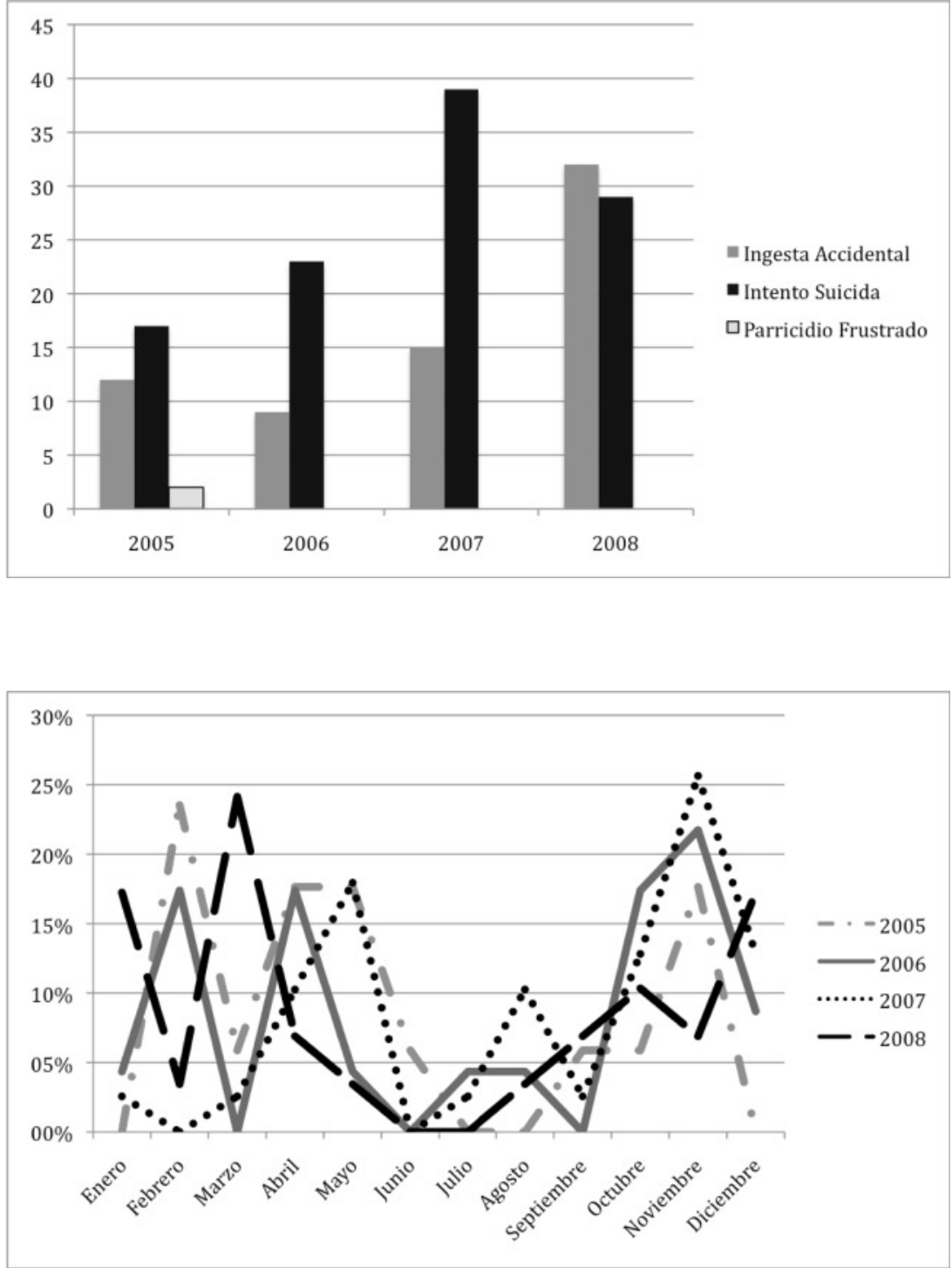

Figura 1. Pacientes egresados de UCI con diagnóstico de intoxicación por fármacos con intento suicida.
Figura 2. Distribución estacional de pacientes con intoxicación por fármacos con intento suicida es mayor en primavera y otoño y menor en invierno $(\mathrm{p}<0,05)$. 
dosis extremadamente altas ( 1 caso con más de $100 \mathrm{~g}$ ). La mezcla de fármacos con gases (monóxido de carbono) estuvo presente en 1 caso.

Complicaciones secundarias se objetivaron en 24 casos; 18 con insuficiencia respiratoria, 5 con arritmias y/o alteraciones electrocardigráficas transitorias y 1 falla hepática.

La estadía promedio en UCI fue de 1,5 días (158 días totales de UCIP, 4 pacientes con más de 3 días). Dieciocho pacientes (16,7\%) requirieron apoyo con ventilación mecánica, 16 por depresión respiratoria transitoria.

Un cuadro psiquiátrico de base fue establecido por especialistas de salud mental, pre o post episodio, en el 92,6\% (100 pacientes), en 55 de ellos $(50,9 \%)$ fue depresión. Existía patología psiquiátrica en la familia cercana en 37 casos $(34,3 \%)$.

El factor asociado más frecuente, mencionado por los pacientes como desencadenante del hecho, fue la disfunción familiar en 81 casos $(75 \%)$. Esto incluyó violencia intrafamiliar, suicidios e intentos de ello, alcoholismo y/o drogadicción parental, desordenes psiquiátricos, ausencia o muerte de alguno de los padres. Se invocaron problemas escolares evidentes en 26 casos $(24,1 \%)$, abuso sexual o violación en 12 casos $(11,1 \%)$, todos por familiares directos o amigos cercanos de la familia. Matonaje fue el factor asociado directo en 6 pacientes $(5,6 \%)$, trastornos de la conducta alimentaria y embarazo 2 casos cada uno $(1,9 \%)$. Más de un factor es reconocible en la mayoría de los pacientes (tabla 2).

No hubo fallecidos en este estudio.

\section{Discusión}

Este estudio es concordante con la literatura en relación a la existencia gravitante y persistente de este problema sanitario de orden biopsicosocial en niños y adolescentes, de difícil solución por la multiplicidad de factores sociales involucrados. La familia como factor de apoyo en el desarrollo armónico del niño y adolescente aparece como el elemento disfuncionante asociado más relevante. La alta incidencia de trastornos de salud mental se agrega como eventual factor predisponente.
Tabla 1. Fármacos utilizados en intoxicación por fármacos con intento suicida

\begin{tabular}{|lcc|}
\hline Tipo droga & Pacientes & \% \\
\hline Polifarmacia & 57 & 57,0 \\
Monofármaco & 51 & 43,0 \\
Antidepresivos & 45 & 41,0 \\
Sedantes & 53 & 49,0 \\
Analgésicos/antiinflamatorios & 14 & 12,9 \\
Tóxicos & 5 & 4,6 \\
Otros & 48 & 44,0 \\
\hline
\end{tabular}

Tabla 2. Factores asociados en intoxicación por fármacos con intento suicida

\begin{tabular}{|lcc|}
\hline Factores asociados & $\begin{array}{c}\text { n de } \\
\text { pacientes }\end{array}$ & $\begin{array}{c}\text { \% de } \\
\text { pacientes }\end{array}$ \\
\hline Desorden psiquiátrico & 100 & 92,6 \\
Disfunción familiar & 81 & 75,0 \\
Problemas escolares & 26 & 24,1 \\
Abuso o violación & 12 & 11,1 \\
Alcohol/drogas & 8 & 7,4 \\
Bullying & 6 & 5,6 \\
Obesidad & 5 & 4,6 \\
Discusión familiar & 4 & 3,7 \\
Embarazo & 2 & 1,9 \\
Trastorno conducta alimentaria & 2 & 1,9 \\
\hline
\end{tabular}

Chile, país en desarrollo, tiene factores de riesgo especiales, a pesar de una mejoría constante en sus indicadores de salud y de calidad de vida. El hoy fácil acceso a la información, pone aún más en evidencia las diferencias socioeconómicas, con aspiraciones difíciles de cumplir ${ }^{2-4}$. La nueva estructura de la familia, mono o biparental, tratando de cumplir con expectativas financieras, deja a los niños y adolescentes solos con sus problemas.

La frecuencia de intento de suicidio en nuestro estudio y en la literatura se mantiene e incluso aumenta, dependiendo de realidades diferentes, del grupo etáreo y variando en la modalidad elegida. La estacionalidad es significativa. Puede estar asociada al alza de depresiones en primavera y otros factores a estudiar. 
Importante a ser considerado para el desarrollo de planes de prevención futuros. Llama la atención la discrepancia entre las cifras del MINSAL $^{15}$ en relación a mayor frecuencia en niños que niñas, comparado con el estudio epidemiológico chileno recién publicado ${ }^{16}$ y nuestros resultados, que evidencian lo contrario. Esto se podría deber a que los hombres mueren más, hecho no observado en nuestra serie.

Comparado con un estudio chileno previo ${ }^{3}$, la gravedad estaría aumentando al requerir en los últimos años ingreso a UCI por la cantidad y calidad de fármacos ingeridos. El estudio revela que muchos fármacos, teóricamente de acceso restringido, están presentes en los hogares en grandes cantidades, sobre todo antidepresivos, benzodiazepinas, antihipertensivos y antiinflamatorios. No fue excepcional que los pacientes fueran admitidos con un elevado número y mezcla de comprimidos ingeridos.

Un $30 \%$ de los pacientes, en su mayoría niñas, tenían un intento previo aumentando el riesgo de lograr el objetivo finalmente, como se reporta en la literatura ${ }^{10}$. Esta reincidencia evidencia que los factores de riesgo asociados no fueron resueltos, el soporte y manejo fue insuficiente y que se requiere de un mayor esfuerzo integral ${ }^{19-21}$.

Cien de 108 pacientes tenían un desorden psiquiátrico de base. Esto sumado a los factores asociados de riesgo similares a los reportados, predominantemente ausencia o insuficiente apoyo en casa, engloban el escenario propicio para un intento suicida en este grupo de niños de diferentes edades, en un amplio rango entre $\operatorname{los} 7$ a los 16 años.

Victimización y victimario de matonaje o bulllying son factores de riesgo tanto de depresión como para intentos suicidas en la niñez y la adolescencia. En este estudio el bullying como causa de intento suicida es aún bajo en comparación con otras realidades ${ }^{7}$. Similar hallazgo se observa en relación a trastornos de conducta alimentaria ${ }^{14,17}$.

Para los casos de abuso y violaciones, es trascendente que sólo miembros de la familia fueron responsables de estos actos, aumentando el riesgo y la soledad de los niños y su dificultad de poder resolver estas situaciones.
Más de un factor de riesgo asociado estuvo presente en casi todos los pacientes haciendo difícil saber cuál fue el gatillante mayor.

A pesar de que no hubo fallecidos, que el requerimiento de ventilación mecánica invasiva fue sólo transitorio, que las estadías en UCIP cortas, mayoritariamente de bajo costo y "fáciles" desde el punto de vista técnico-clínico, el impacto por esta patología en el equipo de salud es alto pero difícil de medir. Los complejos problemas biopsicosociales subyacentes se asociaron al sentimiento de no resolución del problema básico por parte del equipo de salud.

\section{Conclusiones}

Los ingresos a nuestra UCIP debido a intoxicación por fármacos con fines suicidas han aumentado en estos últimos años, causando un incremento de hospitalizaciones breves pero indispensables, sin mortalidad en este estudio.

La mayoría de los pacientes fueron niñas, adolescentes, con importante disfunción familiar, con múltiples factores de riesgo asociados, en familias con abundantes fármacos disponibles en casa, con una patología de salud mental basal identificada en casi todos los casos.

Se verificó la asociación de intento de suicidio con una alta prevalencia de trastornos psiquiátricos de base.

Se requiere a futuro de un estudio detallado de los factores asociados y desencadenantes para el desarrollo de planes de prevención y manejo adecuado.

\section{Referencias}

1.- Silva L: Intento de Suicidio, Cap V. Sección de Psiquiatría Fundación Santa Fe de Bogotá; 1334-8.

2.- AACAP Official action. Practice parameter for the assessment and treatment of children and adolescents with suicidal behavior. J Am Acad Child Adolesc Psychiatry 2001; 40 (7 Suppl ): 24S-51S

3.- Leiva H, Alamos L, Prüssing L, Uriarte A: Intento de Suicidio: características clínicas y epidemiológicas. Sexta Región de Chile 2002-2004. An Pediatr (Barc) 2008; 69 (2): 110-4.

4.- Tuesca R, Navarro E: Factores de riesgo asociados al 
suicidio e intento de suicidio. Salud Uninorte Barranquilla (Col) 2003; 17: 19-28.

5.- Suominen K, Lönnqvist J: Determinants of Psychiatric hospitalization after attempted suicide. General Hospital Psychiatry 2006; 28: 424-30.

6.- Miranda R, Scott M, Hicks R: Suicide attempt characteristics, diagnoses, and future attempts: comparing multiple attempters to single attempters and ideators. Am. Acad. Child Adolesc. Psychiatry 2008; 47 (1): 3240.

7.- Brunstein A, Marrocco F, Kleinman M: Bullying, Depression, and Suicidality in Adolescents. J Am Acad Child Adolesc Psychiatr 2007; 46 (1): 40-9.

8.- Insel B, Gould M: Impact of Modeling on Adolescent Suicidal Behavior. Psychiatr Clin N Am 2008; 31: 293 316.

9.- Jacobs D, Baldessarini C: Practice Guidelines for the Assessment and Treatment of Patients with Suicidal Behaviors. American psychiatric association steering committee on practice guidelinesps.

10.- Miranda R, Scott M: Suicide Attempt Characteristics, Diagnoses, and Future Attempts: Comparing Multiple Attempters to Single Attempters and Ideators. Am Acad Child Adolesc Psychiatry 2008; 47 (1): 32-40.

11.- Greydanus D, Calles J: Suicide in Children and Adolescentshi Prim Care Clin Office Pract 2007; 34: 259-73a.

12.- Valdivia M, Ebner D: Hospitalización por intento de suicidio en población pediátrica: una revisión de 4 años. Rev Chil de Neuro-Psiquiat 2001; 39: 211-8.
13.- López-Garcia A, Serradilla C: Intentos de suicidio: revisión de 23 casos. An Pediatr (Barc) 2002; 56: 199-204.

14.- Hawton K, Townsend E: Deliberate self harm: systematic review of efficacy of psychosocial and pharmacological treatments in preventing repetition. BMJ 1998; 317: 441-7.

15.- www.MINSAL.cl. Primer borrador Objetivos Estratégicos Plan Nacional de Salud 2011-2020: 108.

16.- Ventura-Juncá R, Carvajal C, Undurraga S: Prevalencia de ideación e intento suicida en adolescentes de la Región Metropolitana de Santiago de Chile. Rev Med Chile 2010; 138: 309-15.

17.- Suominen K, Isometsa E: Substance abuse and male gender as risk factors for deaths and suicide: a five year follow- up study after deliberate self-harm. Soc Psychiatry Psychiatr Epidemiol 2004; 39: 720-4.

18.- Hepp U, Moergeli H: Attempted suicide factors leading to hospitalization. Can J Psychiatry 2004; 49: 736-42.

19.- Jacobs D, Baldessarini R: Conwell Practice Guideline for the Assessment and Treatment of patients with Suicidal behaviors. Work Group of Suicidal Behavior 2003; www.psych.org 1

20.- Gould M, Marroco F, Kleiman MA: Evaluating iatrogenic risk of youth suicide screening programs. A randomized controlled trial. JAMA 2005; 43: 1635-43.

21.- Kitainik $D$ : No fue iatrogenico indagar sobre ideación suicida en adolescentes. Evidencia-Actualización en la práctica pediátrica 2006; 9 (2): 45. 